\title{
Impact of Auditory Verbal Therapy in Children with Cochlear Implant
}

\author{
Nikita Chatterjee, ${ }^{1}$ Indranil Chatterjee, ${ }^{1}$ Aditya Sarkar ${ }^{1}$
}

\begin{abstract}
$\underline{\text { Introduction }}$
Auditory Verbal Therapy (AVT) is considered as most successful therapeutic approach for cochlear implant children. The impact of auditory verbal therapy has been studied vividly in Indian context. The number of cochlear implantees receiving AVT has been increased nationwide but there is no structured protocol that is used to assess the efficacy of AVT on children with cochlear implant. Hence, it is important to develop a structured protocol consisting of established tools to study the effectiveness of AVT. Materials and Methods

The study was conducted on 62 (male-38, female-24) bilaterally profound sensorineural hearing impaired children with cochlear implant done under ADIP (Assistance to Disabled Persons for Purchase/ Fitting of Aids and Appliances) scheme. Pre-therapy assessments were done before 2 weeks of cochlear implantation using Functional Auditory Performance Indicator (FAPI), Assessment of language development, Speech intelligibility rating scales, Brown's (1973) stages of mean length of utterance $(M L U)$ assessment, and Overall rating of parental satisfaction on a visual analog scale of 0 to 10. Auditory verbal therapy (AVT) was given for 6-8 months, twice per week under video-monitoring of the sessions. Post therapeutic scores were obtained by assessing with the same tools through observation and interaction with the children and interviewing the caregivers.

$\underline{\text { Results }}$

Independent Sample's T-test in SPSS -16 software were used and significant differences between pre and post therapy scores were obtained $(p<0.005)$.

Conclusion

These tools can be used further as a protocol for assessment of baseline skills before AVT, then develop an individualized management program and finally to assess the improvement after AVT.

Kevwords

Auditory Verbal Therapy; Cochlear Implants
\end{abstract}

ABSTRACT

$\mathrm{A}$ uditory Verbal Therapy (AVT) is a parentoriented early intervention approach used for hearing impaired children, first coined by Ling, Beebe, and Pollack. ${ }^{1}$ It involves individualized diagnostic sessions emphasizing the use of hearing technology in optimal and meaningful conditions hence emphasizes on developing hearing as an active sense. The auditory verbal therapist follows the principles of auditory verbal therapy under the guidelines of ADIP (Assistance to Disabled Persons for Purchase/ Fitting of Aids and Appliances)

1 - Department of Speech and Hearing, Ali Yavar Jung National Institute of Speech and Hearing Disabilities (Divyangjan), Kolkata

\section{Corresponding author:}

Dr Indranil Chatterjee

email: inchat75@gmail.com scheme adapted by principles originally developed by Pollack and "rigorous application of techniques, strategies, conditions and procedures that facilitate and promote optimal acquisition of spoken language through listening". The listening environment for AVT can be enhanced by various ways like therapist sitting in front of child and using techniques like acoustic highlighting technique, pausing, providing alternatives. A certified auditory verbal therapist may continue as a part of the child's educational team helping the child to progress through inclusion in regular listening environment and social platform from the early childhood onwards.

Since, it has been the constant endeavor of the Government to provide the disabled persons with aids/ appliances, essential for their rehabilitation, at minimum costs, The Ministry of Social Justice and Empowerment, Govt. of India has introduced one such scheme of 
Assistance to Disabled Person for Purchasing/Fitting of Aids and Appliances - "ADIP-Scheme" which includes cochlear implant and post-operative rehabilitation along with mapping schedule. Under the ADIP Schemerevised (2014), the AVT that has been provided to cochlear implant beneficiaries without any cost for 1 year has been now extended to 2 years with mapping.

The cochlear implant has been found to be beneficial for patients with severe to profound, steeply sloping type hearing loss; who do not get benefitted adequately with the hearing aids but have an intact auditory nerve. A detailed assessment protocol of the child has to be done before deciding whether the child is a suitable candidate for cochlear implant, provided by ADIP scheme, administered by a team of professionals which includes audiologist, speech and language pathologist, ENT surgeon, paediatrician, neurologist, etc. Approximately after 2 to 3 weeks of surgery, the implant and the external processor are programmed and mapped for first time by an audiologist, followed by which, the role of speech and language pathologists began by providing post-operative speech and language rehabilitation to the implantees.

The impact of auditory verbal therapy has been studied vividly in Indian context. After advent of ADIPscheme, the number of cochlear implantees has been increased nationwide at the rate of 500 implants per year. Hence, there is a wide need to study the effectiveness of AVT on listening and linguistic performance of children after cochlear implantation under ADIP scheme. No such categorized protocols were used so far to tract the listening and linguistic skills of the cochlear implantees. So, a structured protocol using established tools has to be conceptualized with reference to listening and speech perception, linguistic, speech production skills and overall parent's satisfaction from AVT.

\section{Materials and Methods}

This prospective observational study was done at the institute, where the authors are attached. Sixty two participants (male-38/female-24) were selected within the age range of 2.5 to 7.5 years (mean- 5.53 and S.D. \pm 1.21 ) each having bilateral severe to profound sensorineural hearing loss (pure tone average $>70 \mathrm{dBHL}$ ) with congenital onset and normal cognitive, social and motor milestones. Cochlear implantation was done under ADIP scheme in which 32 participants using DIGISONIC $®$ cochlear implant and 30 participants using NUCLEUS $\AA$ (by COCHLEAR ${ }^{\circledR}$ ) cochlear implant.

All the participants were selected as per ADIP scheme-R guidelines for AVT. They received cochlear implant before 5 years (as per the ADIP guidelines) and received mapping on 1st, 2nd, 4th, 6th, 10th, 14th and 18 th week post switch-on and once in 3months if required afterwards. Both the parents and other caregivers were counseled to use their native language with their children. Irregular participants with inactive parental participation for whom the transfer of skills to parents and home management was difficult were excluded.

The tools used for the pre and post therapy assessment included Functional Auditory Performance Indicator (FAPI), Assessment of language development, Speech intelligibility rating scales, Brown's (1973) stages of MLU assessment and the Overall rating of parental satisfaction on a visual analog scale of 0 to 10 .

The Functional Auditory Performance Indicators (FAPI) was developed by Brown and Johnson (2003) to assess the functional auditory skills of the children with hearing loss. It is used by parents, therapist, early interventionists and teachers. ${ }^{2}$ The profile lists the auditory skills in an integrated hierarchical manner. There are seven categories in the checklist: - sound awareness, sound is meaningful, auditory feedback, localizing the sound, auditory discrimination, shortterm memory, linguistic-auditory processing. A four tired scoring paradigm has been created. (Table I)

Assessment of language development (ALD) developed by Lakkanna, Venkatesh and Bhatt (2010) is a criterion referenced diagnostic tool to measure language development in children from birth to 7.11 years covering both receptive and expressive language skills. ${ }^{3}$ Precursors to language development like attention, vocal development are also embedded in the test. Different aspects of language like vocabulary, syntax, semantics as well thinking/reasoning skills are 
Table I: A four tired scoring paradigm of FAPI

\begin{tabular}{|c|c|c|}
\hline LEVEL OF ATTAINMENT & CORRESPONDING OCCURRENCE & VALUE GIVEN \\
\hline The skill is not present & $(\mathrm{NP})=\mathbf{0 - 1 0} \%$ Occurrence & (score value $=\mathbf{0})$ \\
\hline The skill is emergent & $(\mathrm{E})=\mathbf{1 1 - 3 5 \%}$ Occurrence & (score value $=\mathbf{1})$ \\
\hline The skill is in process & $(\mathrm{P})=\mathbf{3 6 - 7 9 \%}$ Occurrence & (score value $=\mathbf{2})$ \\
\hline The skill is acquired & $(\mathrm{A})=\mathbf{8 0 - 1 0 0 \%}$ Occurrence & (score value $=\mathbf{3})$ \\
\hline
\end{tabular}

included in the assessment. The test material contains the questionnaires and standardized pictures. The test items are divided into items for receptive language (1 to 52) and expressive language (1 to 52) separately. The administration timing of the test ranges from 20-40 minutes. Then the starting point is established below one year of the chronological age. Each test item has specific criteria for passing. Score 1 for passing and score 0 for not and these results are entered in the scoring sheet. Criteria for basal point is obtained when the child passes 3 consecutive items and ceiling point is obtained when the child fails to answer 5 consecutive items and the testing should be stopped there. After testing, the raw score is calculated by subtracting the number of zeroes from the item number of last test item. This raw score is used as appropriate marker to indicate criteria met/ not met.

The Speech Intelligibility rating scale was developed at Ali Yavar Jung National Institute of Speech and Hearing Disabilities (AYJNISHD); Mumbai (2003) is a perceptual scale to see how much the speech is intelligible to the listener on a 7-points scale of 0 to 6 , where 0 implies normal and 6 implies poor intelligibility. (Table II) This is done at different levels that are at word level, phases, sentences and conversational speech, depending on the patient's receptive and expressive vocabulary.

Brown's stages of Mean length of utterance (MLU) assessment is a strong communication marker to understand language development in children. ${ }^{4}$ Five stages of language development based on the MLU are shown in Table III.

Table II: The seven points rating of the Speech Intelligibility Rating Scale

\begin{tabular}{|c|l|}
\hline SCORE & \multicolumn{1}{c|}{ INTERPRETATION } \\
\hline 0 & Normal \\
\hline 1 & Can understand with little effort; however feel speech is not normal \\
\hline 2 & Can understand with little effort occasionally need to ask for repetition \\
\hline 3 & Can understand with concentration and effort specially be sympathetic listener \\
\hline 4 & Can understand with difficulty and concentration by family, but not others \\
\hline 5 & Can understand with effort if content is known \\
\hline 6 & Cannot understand at all even when content is known \\
\hline
\end{tabular}


Table III: Five stages of MLU development designed by Brown

\begin{tabular}{|l|c|c|}
\hline \multicolumn{1}{|c|}{ STAGES } & MLU & APPROXIMATE AGE (IN MONTHS) \\
\hline 1. Semantic roles and syntactic relations & $1.0-2.0$ & $12-26$ \\
\hline 2. Grammatical morphemes and modulating meaning & $2.0-2.5$ & $27-30$ \\
\hline 3. Modalities of simple sentences & $2.5-3.0$ & $31-34$ \\
\hline 4. Embedding & $3.0-3.75$ & $35-40$ \\
\hline 5. Co-ordination & $3.75-4.5$ & $41-46$ \\
\hline
\end{tabular}

An 11 visual analog rating scale (from 0 to 10 point) was developed in AYJNISHD, RC-Kolkata, to track the rate of parental satisfaction from the cochlear implant and post-implant rehabilitation are scored in accordance. The scoring pattern is such that, 0 stands for no satisfaction at all, 5 stands for medium satisfaction while 10 stands for tremendously satisfied.

The study procedure is divided into 5 phases. First, children who were best fitted as per the selection criteria were chosen for the study and their parents and caregivers were given information about the study and its importance and were requested to sign a consent form that pledged for their permission and cooperation for the study. In the second phase pre-therapy assessment scores were obtained for listening skills using Functional Auditory Performance Indicator, Linguistic skills using Assessment of language development, Speech production using Speech intelligibility Rating Scales and Mean Length Utterance were obtained by administering the test using the materials required to administer the test and in specific test environment, before 2 weeks of cochlear implantation. In the third phase, auditory verbal therapy was given for 6-8 months, twice a week by the informally trained auditory verbal therapist, either the post-graduate students or the interns. The scheduled and individualized therapy plan for each participant were prepared and the goals were set to improve the listening skills (sound awareness and detection, sound localization, auditory discrimination and auditory comprehension), linguistic skills (receptive and expressive language skills), speech production (phonetic and phonological skills) and mean length of utterance of morphemes. The overall duration of therapy session was 45 minutes in which included parental counseling and guidance for home management. (Table IV)

\section{Results}

The findings of pre and post therapy scores of auditory skills were compared using Independent Sample's T-test in SPSS-16 software showing significant difference was found $(p<0.05)$ between pre and post therapy scores of the auditory skills in Functional Auditory Performance Indicator (FAPI). (Fig. 1)

The findings of pre and post therapy scores of Receptive Language assessment and Expressive Language assessment were compared using Independent Sample's T-test in SPSS-16 software. Significant difference was also found $(p<0.05)$ between pre and post therapy scores of receptive and expressive language skills tested by Assessment of language development. (Fig. 2)

The findings of pre and post therapy scores of Speech Intelligibility also found to have significant difference $(p<0.05)$ between pre and post therapy scores of Speech Intelligibility Rating Scale. (Fig. 3)

The pre and post therapy scores of Mean Length of Utterance also showed significant difference $(\mathrm{p}<0.05)$ between pre and post therapy scores of Mean Length of utterances. (Fig. 4) 
Table IV: Auditory verbal therapy programme

(a) Improving the Auditory Skills: It included improving of Awareness of sound, Supra-segmental discrimination and association, Segmental discrimination and association, Identification and Processing and comprehension.

(b) Developing Speech Skills: It included improving Pre-speech (Imitating two different vocal lengths), Isolation (vowel and consonant in babble in isolation), Vocal play and sound sequencing (to facilitate vocal play and to remediate sound sequencing), Words (to facilitate production of 5 words with $80 \%$ intelligibility and to facilitate production of at least 25 words with $80 \%$ intelligibility), Phrases (to facilitate production of phrases with $\mathbf{8 0} \%$ intelligibility) and Sentences (to facilitate spontaneous production of sentences with 5-6 words with 80\% intelligibility and with $7+$ words with $80 \%$ intelligibility).

(c) Developing Language Skills: It included improving of Word approximation (to facilitate vocalization to gain attention), Word production (to facilitate production of true words), Connected utterances/ basic sentence production (to facilitate production of 2 word phrases), Connected utterances (to facilitate production of 10 different 2-3 word sentences), Simple sentences with grammatical markers (to facilitate production of simple sentences with grammatical structures), Expanded sentences (to facilitate production of interrogative sentence formulation and expanded sentence formulation) and Complex sentences (to facilitate production of conjoining sentence, complex sentences and narration).

(d) The fourth phase included obtaining the post therapeutic scores by assessing the post therapeutic status of the children administering with the same set of tools used for assessing specific area or skill, that were used during the pre-therapy period. Lastly compilation of the pre and post therapy data were done on Microsoft excel-sheet. These data were then compared to find out significant difference between the pre and post therapy using Independent Sample's T-test in SPSS-16 software.

The pre and post therapy overall satisfaction ratings given by parents after Auditory Verbal Therapy were also compared. Significant difference between pre and post therapy overall satisfaction ratings given by the parents in the visual analog scale were good (mean $=8.06$; S.D. \pm 0.73 ) for their child's improvement in respect to speech-language and hearing skills after auditory verbal therapy, which was significantly better than the ratings given by parents before the auditory verbal therapy i.e., somewhat satisfied (mean=4.05; S.D. \pm 1.24$)$. (Fig. 5)

\section{Discussion}

The objectives of the study were to find out and compare the listening skills, linguistic skills, speech intelligibility,

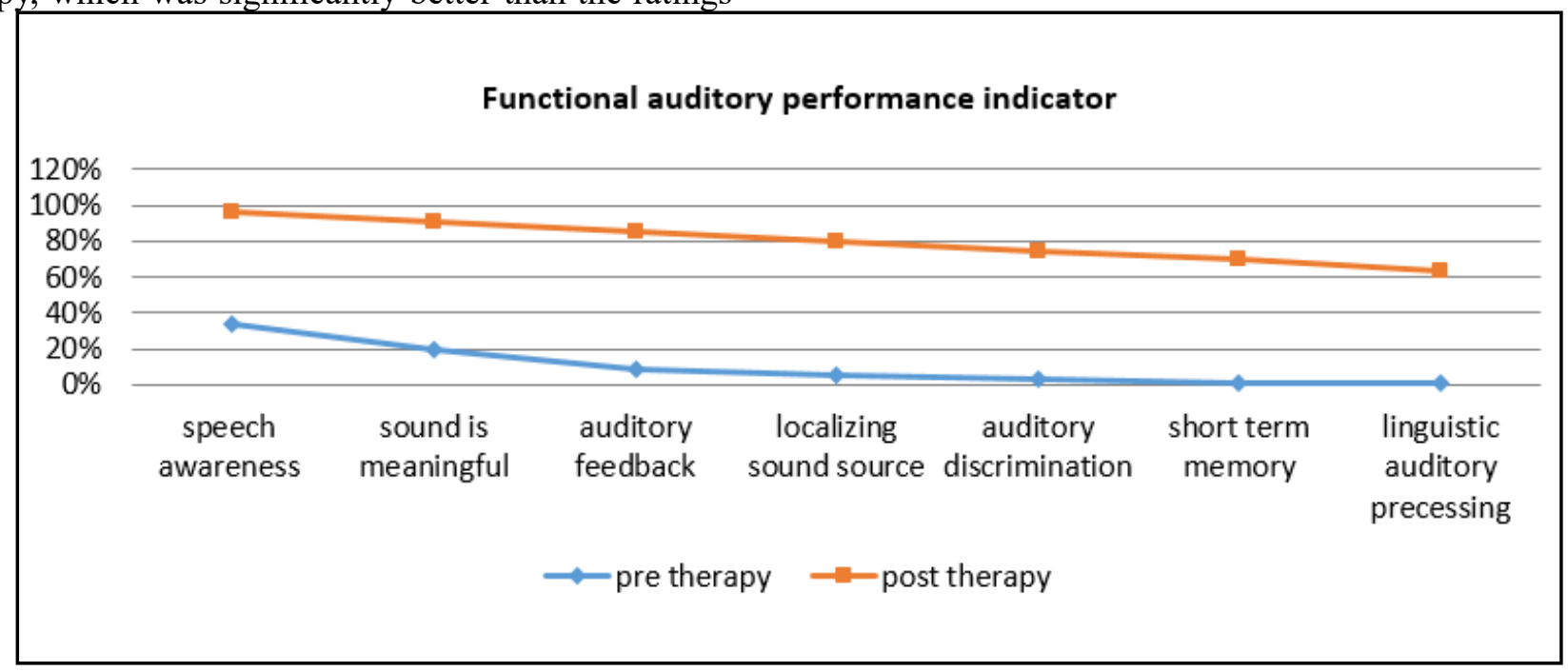

Fig. 1. Comparison of pre and post therapy FAPI scores 


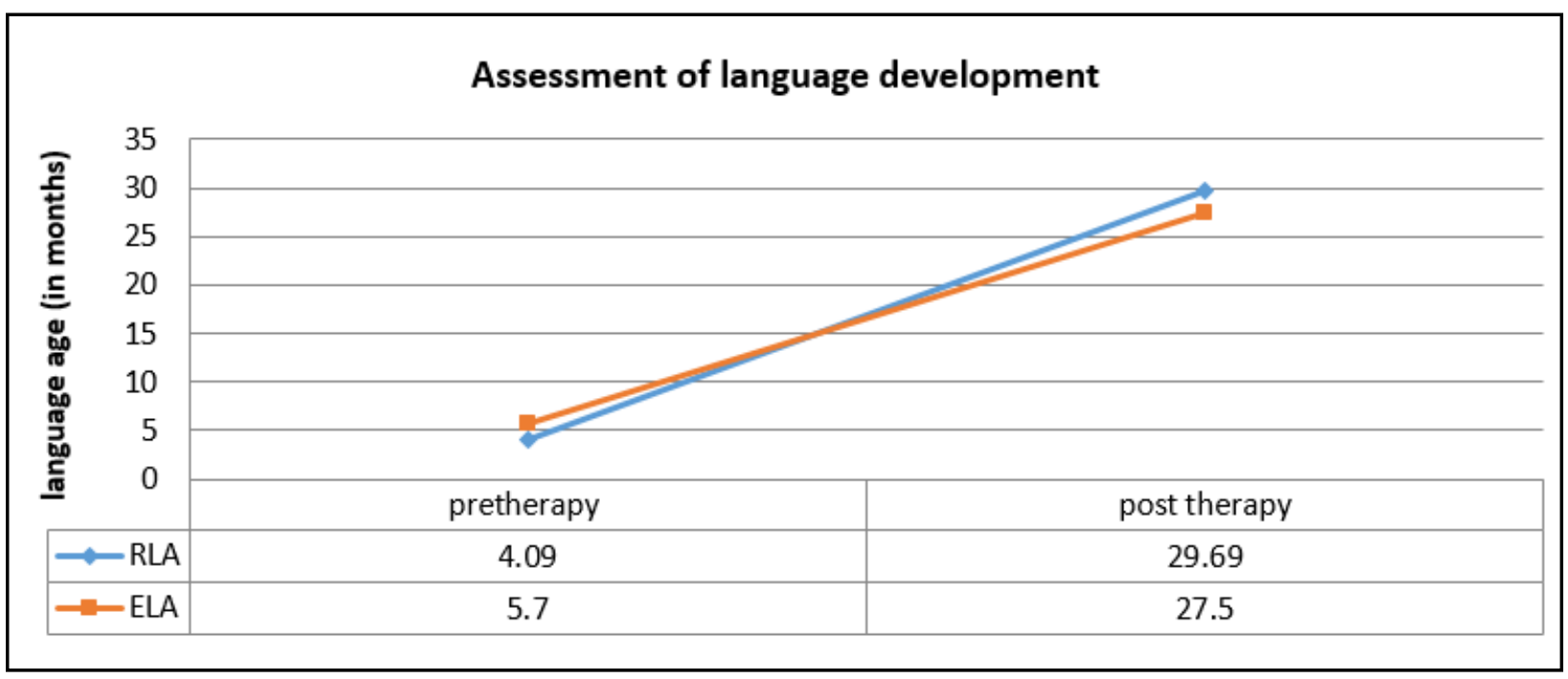

Fig. 2. Comparison of Pre and Post therapy Receptive and Expressive Language scores obtained by assessment of language development

mean length of utterances of morphemes and to rate the overall parental satisfaction of parents from the cochlear implant and compare with post auditory verbal therapy rating.

This preliminary study reveals that although, there was a significant difference in pre and post therapy scores, none of the participants achieved age appropriate speech and language skills however children implanted at early age, have more improvement than others. The differences between the pre and post therapy scores of the listening skills on functional auditory performance checklist suggested significant improvement in listening skills, within 6-8 months of cochlear implantation. For linguistic skills, the differences between the pre and post therapy scores for receptive and expressive language skills on Assessment of Language Development (ALD) suggests, there is significant improvement in their receptive and expressive language skills within 6 months. The mean scores of overall intelligibility of speech in the post-therapy assessment had a significant

\section{Speech intelligibilty rating scale}

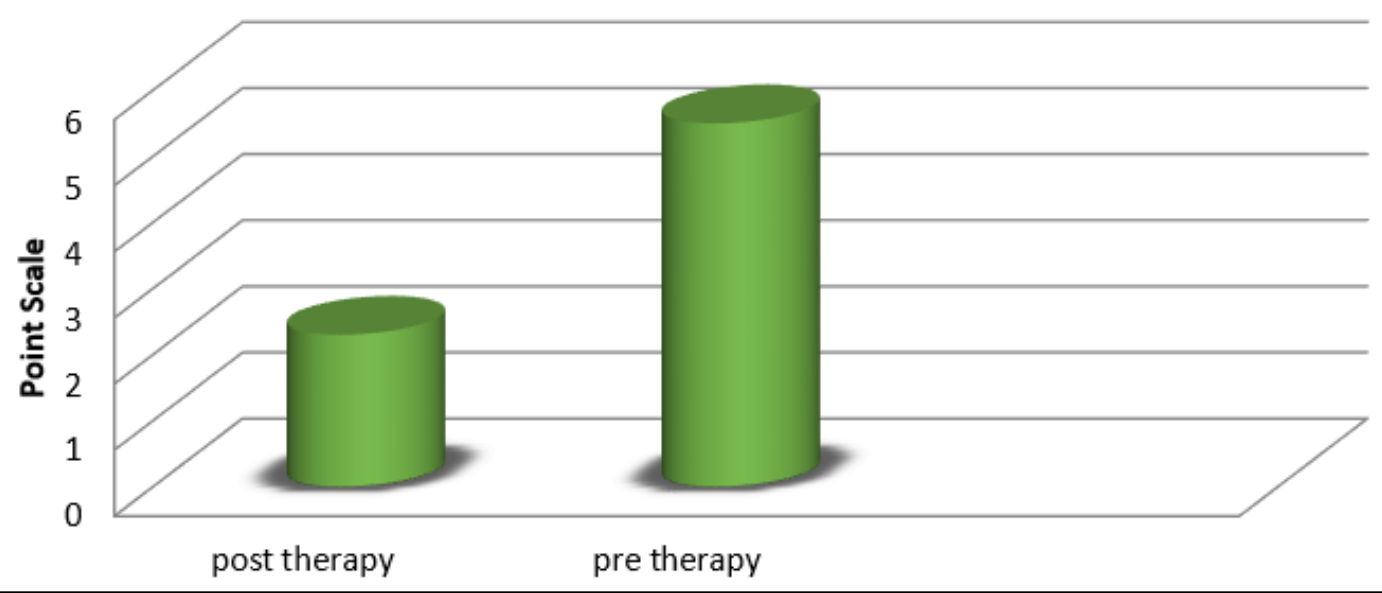

Fig. 3. Comparison of Pre and Post therapy scores obtained by Speech Intelligibility Rating Scale. 


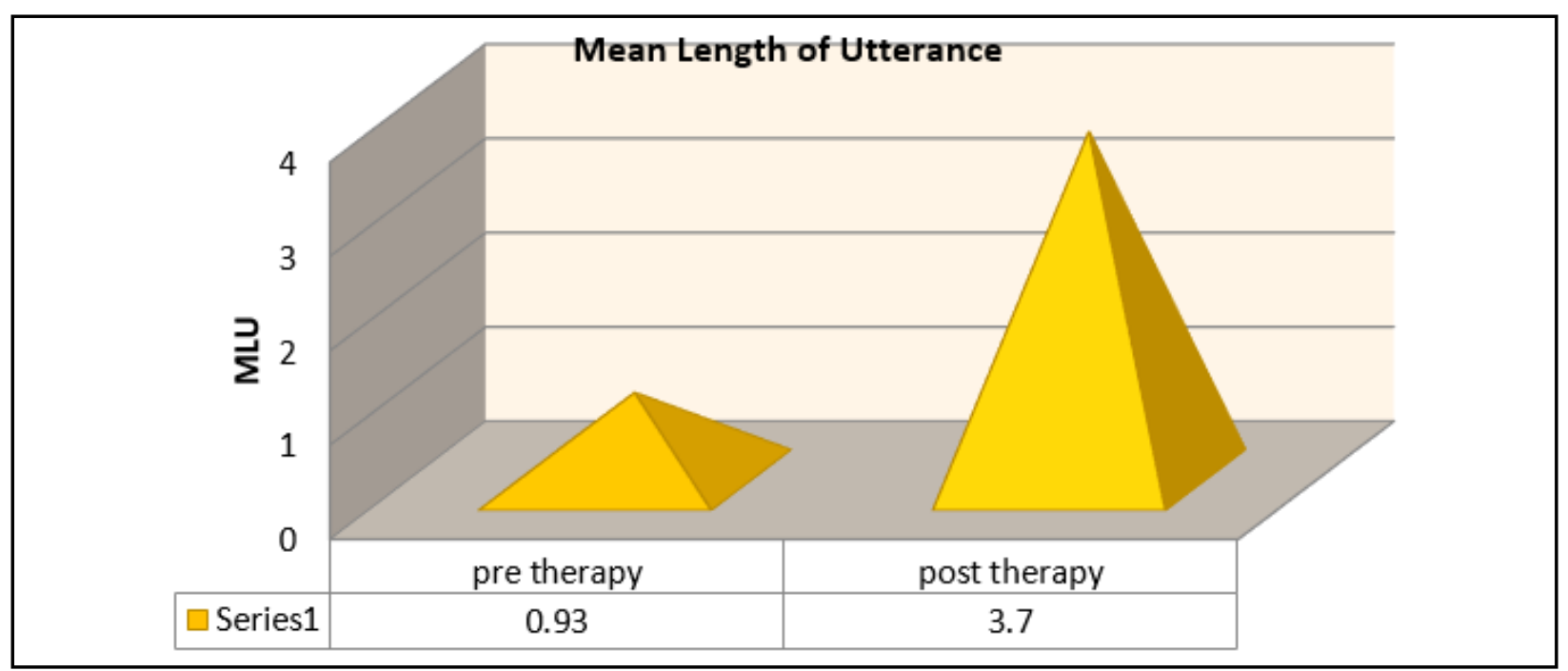

Fig. 4. Comparison of Pre and Post therapy scores obtained by Brown's (1973) stages of MLU assessment

difference from the pre-therapy ratings but the perception of supra-segmental features in speech and the voice of the cochlear implant children had significant but not much stronger outcomes. Similarly, for the mean length of utterances, it has been found that although, there is significant increment in the mean length of utterance of the participants; they still have significantly delayed mean length of utterances in morphemes. It is important to explore the parental expectation and satisfaction with the implant habilitation, parental stress, and quality of life of children and their families, especially due to the diversity in the cultural differences and socio-economic status in the Indian context, which was rated on a visual analog rating scale showing the satisfaction level and expectancy of parents after the implant and therapy is significantly higher.

A recent study conducted by Nadurkar and Susmitha to see the progress in listening skills of cochlear implant children suggests development of auditory skills triggers in first three months of cochlear implantation. ${ }^{5}$ A study

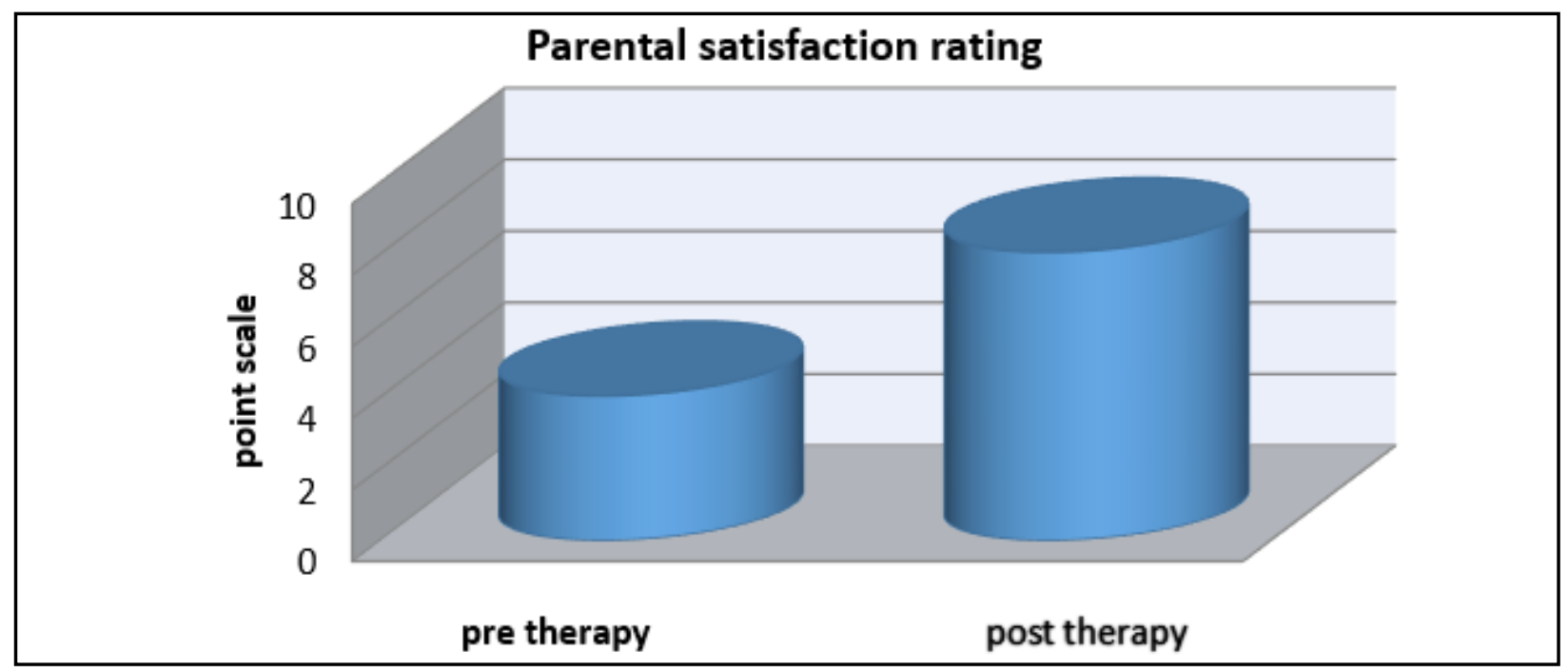

Fig. 5. Comparison of Pre and Post therapy overall satisfaction ratings given by parents after Auditory Verbal Therapy after cochlear implantation. 
conducted by Dornan et al. suggest that within nine months of auditory verbal therapy, there is a significant boost in linguistic skills of cochlear implant children and age appropriate linguistic skills can be achieved within 24 months. ${ }^{6}$

Most and Peled in his study concluded that perception of supra-segmental features of speech in children with cochlear implant had not a significant effect on children with hearing aids. ${ }^{7}$ Mahmoudi et al. studied on voice abnormalities of cochlear implant children, suggested that "voice abnormalities in speech of cochlear implant children had no significant difference from the children using hearing aids". ${ }^{8}$ In dissonance to these findings, Tyler et al. concluded in his study that use of cochlear implant has been associated with stronger outcomes in speech production and verbal intelligibility compared with children using conventional hearing aids. ${ }^{9}$

For the variability in these results, many factors seem to contribute such as the effect of environment and culture, which include gender temperament, socioeconomic status, caregiver interactions, reading habits and social environments. Indeed, with poor socioeconomic background, disparities in the development of language processing are arguably and most consistently found with decrease in vocabulary, phonological awareness and syntax at many different developmental stages which is also supported by Bradley and Corwyn in their study on socioeconomic status and child development. ${ }^{10}$

\section{Conclusion}

Though several studies have been demanded the same kind of findings but this study gives a comprehensive light of knowledge with reference to different formats of evaluation in large number of subjects. Studies even proved efficacy of AVT in Cochlear Implant users with small sample sizes but for better population estimation large sample size is always better. In Indian context, there is no protocol-based study on efficacy of AVT on cochlear implant done on a large number of populations. Hence, this study is found to be useful to form a protocol consisting of scales used to measure the efficacy of AVT and can be used for assessment and management for intervention and hence, for tracking the overall improvement of the child. There are several limitations in this study. The limitations included the socio-economic status, language exposure and parental participation which play vital role and success of auditory verbal therapy and development of listening and linguistic skills in hearing impaired children.

This study created numerous recommendations to be implicated for further research. There is a vivid need to develop a strong protocol to check the efficacy of the cochlear implant and the post-surgical habilitation provided under the government schemes; which should be included and implemented under the government policy. Therefore, this research can throw a light on the implication of amendment of a strong protocol of checking efficacy and further development of a treatment efficacy software for assessment, management and tracking development using this structured protocol.

\section{References}

1. Ling D. Auditory-verbal options for children with hearing impairment: Helping to pioneer an applied science. The Volta Review 1993; 95(3): 205-15

2. Stredler BA, Johnson DC. Functional auditory performance indicators: An integrated approach to auditory development. 2003 Available from: http://www.cde.state.co.us/cdesped/ SpecificDisability-Hearing.htm

3. Lakkanna S, Venkatesh K, Bhat JS. Assessment of Language Development. Bakersfield, CA: Omni therapy services, 2008

4. Brown R. A first language: The early stages. Cambridge Mass: Howard University Press 1973. doi:https://doi.org/10.1017/ S030500090000074X

5. Nandurkar A, Susmitha CG. Listening skill progress in children with cochlear implant in the first three months after implantation. Int J Otolaryngol Head Neck Surg. 2017; 3(3): 632-38

6. Dornan D, Hickson L, Murdoch B, Houston T. Speech and language outcomes for children with hearing loss educated in Auditory-Verbal therapy programs: A review of evidence. Communicative Disorders Review 2007; 2(3-4): 157-72

7. Most T, Peled M. Perception of suprasegmental features of speech by children with cochlear implant and with hearing. Journal of Deaf Student and Deaf Education 2007; 12 (3): 35061

8. Mahmoudi Z, Rahati S, Ghasemi M, Rajati M, Asadpour V, Tayerani H. Diagnosis of voice abnormalities in speech of Children with cochlear implant and Hearing aid with artificial decision making systems. J Med Sci. 2009; 5(2): 67-78 
9. Tyler RS, Teagle HB, Kelsay DR, Gantz BJ, Woodworth GG, Parkinsin AJ. Speech perception by prelingually deaf children after six years of cochlear implant use: Effects of age at implant. Int J Language and Communication Disorders, 2001; 36: 82-4
10. Bradley RH, Corwyn RF. Socioeconomic status and child development. Annual Review of Psychology 2002; 53(1): 37199 